\title{
Civilisations
}

Revue internationale d'anthropologie et de sciences

humaines

$54 \mid 2006$

Expériences de recherche en République

démocratique du Congo

\section{Enquête socio-économique des ménages à Lubumbashi (2002)}

Méthodologie et réflexions

Marcel Remon et César Nkuku

(2) OpenEdition

Journals

Édition électronique

URL : https://journals.openedition.org/civilisations/360

DOI : 10.4000 /civilisations.360

ISSN : 2032-0442

Éditeur

Institut de sociologie de l'Université Libre de Bruxelles

Édition imprimée

Date de publication : 1 avril 2006

Pagination : 95-106

ISBN : 2-87263-006-6

ISSN : 0009-8140

Référence électronique

Marcel Remon et César Nkuku, «Enquête socio-économique des ménages à Lubumbashi (2002) »,

Civilisations [En ligne], 54 | 2006, mis en ligne le 01 avril 2009, consulté le 21 septembre 2021. URL

http://journals.openedition.org/civilisations/360 ; DOI : https://doi.org/10.4000/civilisations.360

(ㄷ) Tous droits réservés 


\title{
Enquête socio-économique des ménages à Lubumbashi (2002) Méthodologie et réflexions
}

\author{
Marcel REMON \& César NKUKU
}

Résumé : L'article décrit la méthodologie suivie lors d'une enquête socio-économique relative aux ménages de Lubumbashi réalisée en août 2002. Cette enquête, financée par la CUD (Commission universitaire pour le développement, une agence des universités francophones de Belgique), a porté sur 13000 ménages, ce qui représente 10\% des ménages dénombrés lors du recensement de 2001. Les étapes de la réalisation de l'enquête sont décrites et analysées, à savoir, la définition des objectifs, la constitution de l'échantillon, la rédaction du questionnaire, la formation des enquêteurs, l'organisation et la réalisation de l'enquête proprement dite, l'encodage des résultats et l'analyse de ces derniers. Des difficultés et des lacunes ont été mises en évidence lors de cette recherche. Elles plaident pour une plus grande disponibilité des chercheurs congolais afin de mieux connaître la littérature scientifique préalablement à l'enquête et de pouvoir se former aux techniques récentes d'analyse statistique et socio-économique. La publication informatisée des données brutes, ainsi que des résultats, peut aider à résoudre ces lacunes.

Mots-clés : recensement, questionnaire, enquête socio-économique, ménages, Lubumbashi.

Summary: This paper describes the methodology used during a socio-economic survey of households in Lubumbashi. Research was carried out in August 2002, financed by the Belgian University Commission for Development (CUD). The survey involved 13,000 families, which represents more than ten percent of all Lubumbashi families. The different steps of the survey are described: definition of objectives, choice of sampling group, drafting the questionnaire, training students, day-by-day organization of the survey, the encoding of the data, and finally, analysis and report writing. The paper also describes difficulties encountered during the research process, emerging largely from time constraints, insufficient knowledge of the foreign experts and poor skills of local researchers. Putting raw data and the final report on the Internet, can help correct such problems in the future.

Key words: census, questionnaire, socio-economic research, households, Lubumbashi. 


\section{Introduction et contexte}

Zn août 2002 eut lieu à Lubumbashi, sous la supervision des deux auteurs de

¿l'article, une vaste enquête socio-économique sur la condition des ménages. Cette recherche a été financée par la Direction Générale de Coopération au Développement (DGCD) dans le cadre de la Commission Universitaire pour le Développement (CUD) du Conseil Interuniversitaire Francophone (CIUF) de Belgique. Cette enquête se déroula quelques mois après un recensement administratif de la population mis en œuvre par les autorités administratives de la Ville.

Avant d'aborder la méthodologie de l'enquête, ainsi que les réflexions critiques qu'il y a lieu de retenir, il est important de situer l'objectif ambitieux de cette enquête. Il s'agissait d'interroger plus d'un dixième de la population recensée, sur des aspects aussi divers que la composition de la famille, les revenus - formels et informels -, les biens matériels des foyers, la fécondité, la scolarité ou l'état de l'habitation. Une telle ambition nécessitait évidemment un investissement important en termes de personnel, ainsi que des ressources financières substantielles et une expertise informatique et statistique.

On aurait pu imaginer un projet plus modeste, mais la rareté de telles enquêtes plaidait pour une réalisation de grande envergure. Cet aspect des choses est important, car il se retrouve dans plusieurs projets de recherche mis en œuvre dans le cadre de la coopération institutionnelle universitaire avec les universités congolaises : la possibilité d'un financement extérieur pour une recherche est souvent l'occasion de réaliser des projets en attente depuis plusieurs années, avec l'objectif de combler le retard accumulé par les institutions en termes d'information ou de publication.

La réflexion conduite dans le cadre de cet article vise à souligner les difficultés rencontrées lors de ce projet. Pour les lecteurs que cela intéresse, les résultats de l'enquête, ainsi que la base de données elle-même, sont accessibles à l'adresse internet $h t t p: / / c u d$. fundp.ac.be/UNILU/enquete_2002.html.

\section{Méthodologie suivie}

Dès que le financement fut approuvé par la Commission Universitaire pour le Développement dans le cadre d'un budget exceptionnel alloué par le Ministère Belge de la Coopération au Développement, une équipe d'experts fut constituée et mise en place en février 2002 pour étudier scientifiquement la faisabilité de cette recherche. Le déroulement de cette activité a été supervisé par les auteurs de cet article. Parmi les experts, il faut citer feu le Professeur Mbuyi Bin Diondo, professeur de géographie de la population, qui a dirigé la sous-commission scientifique de mise en forme finale du protocole d'enquête.

Il s'agit dans ce paragraphe d'indiquer comment a été conçu le protocole d'enquête, comment se sont déroulés les travaux d'enquête sur le terrain et comment a été effectué le dépouillement des données récoltées. Tout cela s'est fait par rapport à des objectifs poursuivis par l'étude. 


\section{Objectifs de l'étude}

Dans le domaine démographique, l'organisation d'un recensement reste onéreuse, tandis que les statistiques recueillies de manière sporadique présentent de nombreuses lacunes. Actuellement on se contente d'extrapolations faites à partir des données de recensements très anciens tels que ceux de 1970 et de 1984 (INS 1993). Depuis lors, aucun autre recensement n'a été organisé. Pour la ville de Lubumbashi, néanmoins, un recensement a été initié en 2000, grâce à un partenariat entre l'Hôtel de ville de Lubumbashi et la ville de Liège. Malheureusement, les variables retenues lors du recensement sont en nombre restreint et ne concernent que l'aspect typiquement démographique, c'est-à-dire le simple dénombrement de la population. L'un des objectifs de l'enquête est de valider, mais aussi de compléter les résultats de ce recensement sur les aspects de la vie sociale de la population qu'il ne livre pas. Il s'agit de produire une image la plus complète possible du panorama de la population et de l'espace urbain de Lubumbashi.

L'enquête socio-économique n'est pas la première à s'inscrire dans cette perspective. Une première grande enquête fut réalisée en 1973 par le Bureau d'Etudes et d'Aménagement Urbain (BEAU) (Houyoux et Lecoanet 1975). Elle fut suivie d'une autre menée, douze années après, en 1985 par l'Institut National de Statistique (INS 1989). En 2000, l'Observatoire de Changement Urbain (OCU) de l'Université de Lubumbashi a réalisé une enquête dans ce sens, mais sur un échantillon de 84 ménages (Petit 2003). La comparaison des résultats de ce travail avec les recherches précédentes occupera une place importante lors de la rédaction du document final.

\section{Constitution de l'échantillon}

Un protocole d'enquête ne peut être conçu que sur base d'un échantillon préalablement bien défini. Ce fut la première préoccupation des experts affectés à la préparation de l'enquête. Pour construire l'échantillon, on s'est référé aux objectifs tels que définis ci-dessus. Il s'est révélé que pour procéder à une bonne vérification d'un recensement, l'échantillon devra rester suffisamment grand et le nombre de variables suffisamment important pour le compléter. Le recensement administratif a indiqué un volume de population totale de 1180387 habitants ainsi que plus de 150000 ménages. Ceci a conduit le comité scientifique de l'enquête à fixer le nombre de ménages enquêtés entre 15000 et 17000 au maximum. Ainsi, l'échantillon serait largement représentatif, soit un dixième de la population et des ménages lushois. L'objectif visé était de couvrir toute la ville (et donc, toutes ses communes) ainsi que toutes les catégories sociales. Au-delà d'une stratification des quartiers dans chaque commune, pour deux quartiers aux morphologies géographiques et sociales identiques, un seul était retenu pour l'enquête. Néanmoins, la priorité était accordée au quartier le plus vaste.

Avec les critères définis ci-dessus, 23 quartiers sur les 42 que compte Lubumbashi ${ }^{1}$ ont été retenus comme secteurs d'enquête. Il s'agit de :

1. Le terme «Lubumbashi » désigne à la fois l'agglomération dans son ensemble, et la commune de Lubumbashi, au centre de la ville. Dans l'article, Lubumbashi désigne généralement la ville de Lubumbashi; nous précisons lorsqu'il s'agit de la commune de Lubumbashi. 


\begin{tabular}{l|l}
\hline \multicolumn{1}{c|}{ Communes } & \multicolumn{1}{c}{ Quartiers } \\
\hline Lubumbashi & Gambela, Kiwele et Makutano \\
\hline Kenya & Luapula et Luvua \\
\hline Kampemba & $\begin{array}{l}\text { Bel air I, Bel air II, Kafubu, Kampemba et } \\
\text { Industriel }\end{array}$ \\
\hline Katuba & Bukama, Kisale, Mwana Shaba, Musumba et Nsele \\
\hline Kamalondo & Kitumaini et Njanja \\
\hline Ruashi & Bendera et Shindaika \\
\hline Annexe & Luwowoshi, Kalebuka, Kimbembe et Kisanga \\
\hline
\end{tabular}

Ce qui précède ne doit pas laisser croire que tous les ménages de ces quartiers ont été investigués. Il a été procédé à un échantillonnage aléatoire au niveau de la rue. Pour chaque quartier désigné, les parcelles à enquêter ont été sélectionnées après un travail de relevé parcellaire. Celui-ci a été réalisé par les enquêteurs à l'issue de leur formation. C'était leur première visite de terrain.

En effet, pendant trois jours, c'est-à-dire du 22 au 24 juillet 2002, les enquêteurs formés, encadrés par des superviseurs, ont sillonné toute l'agglomération pour se rendre compte de la configuration des quartiers à enquêter. Ils étaient répartis en trois équipes, composées de 28 ou 29 membres chacune : la première équipe devait sillonner les communes Kampemba et Ruashi, la seconde celles de Katuba, Kenya et Kamalondo tandis que la troisième est allé dans les communes de Lubumbashi et Annexe. Au cours de ce premier travail de terrain, chaque enquêteur devait remplir des relevés parcellaires pour chaque avenue ou rue qu'il visitait et qu'il parcourait d'un bout à l'autre.

Le travail de relevé parcellaire a permis la détermination des parcelles à enquêter par les organisateurs. L'unité de sondage était la parcelle et tous les ménages rencontrés dans une parcelle devaient être interrogés. Cette dernière option n'a pas été totalement respectée, car il arrivait que, de l'ensemble des ménages dans la parcelle, l'enquêteur ne savait prendre des informations que sur quelques-uns d'entre eux, les autres responsables étant absents lors du (ou des) passage(s) de l'enquêteur.

\section{Définition du protocole d'enquête}

La réflexion sur le protocole d'enquête a nécessité plusieurs séances de travail pour les experts. Finalement, sept rubriques ont été retenues et reprises comme telles dans le feuillet du questionnaire. Chaque thème est décliné en variables disposées en colonnes et auxquelles on a donné des numéros en ordre croissant. Les lignes correspondent aux réponses attendues pour chaque variable.

La première rubrique, «Unité d'habitation », reprend le nom de la commune, du quartier, de l'avenue ou de la rue, le numéro de la parcelle, le nombre de ménages dans la parcelle, le numéro du ménage dans la parcelle et le nombre de personnes dans le ménage. Les " Caractéristiques démographiques des ménages » sont le lien de parenté, le sexe, la date de naissance, le statut résidentiel, l'état matrimonial, le niveau d'instruction, la religion, le type d'activité, la profession principale, la situation dans la profession principale, la profession secondaire, la branche d'activité, la résidence antérieure, la profession dans la résidence antérieure et la date d'arrivée à Lubumbashi. Sous la troisième rubrique, "Fécondité et mortalité au cours des 5 dernières années », on cherche 
à obtenir des données sur la natalité et la mortalité des nourrissons au cours des cinq années qui précèdent l'enquête, car la mortalité des nourrissons est l'un des phénomènes qui caractérisent les villes africaines. Il se pose également à Lubumbashi un problème de tenue de l'état civil. On assiste aujourd'hui à une campagne de sensibilisation en faveur de la déclaration des mariages à l'état civil, l'enquête pourra vérifier le degré d'assimilation de cet appel dans sa rubrique « Nuptialité-Déclaration de mariage à l'Etat Civil ».

L'enquête s'intéresse également aux «langues parlées dans le ménage ». Cette variable peut être révélatrice du niveau d'instruction, mais aussi du visage cosmopolite de la ville.

L'étude s'intéresse d'une façon particulière au « Revenu des ménages ». Parmi les opportunités recherchées se trouve le fait de savoir s'il existe encore en cette période de crise économique aiguë à Lubumbashi des ménages qui recourent à une seule source de revenu pour survivre, le salaire en particulier. On cherche aussi à connaître ce que font les ménages pour pallier à l'insuffisance éventuelle des sources principales de revenu. L'approvisionnement en denrée alimentaire est un autre problème rencontré dans les ménages lushois; il faudra découvrir les voies de sortie auxquelles ces derniers ont recours. Par ailleurs, il s'est aussi avéré nécessaire d'adresser une attention particulière à l'utilisation du revenu et aux dépenses de consommation, ainsi qu'aux biens (équipement) des ménages en tant qu'indicateurs soit de richesse, soit de pauvreté.

Enfin, la septième rubrique concerne la morphologie de l'habitat. Les villes africaines se caractérisent aussi par la précarité de l'habitat et de son environnement. La qualité de l'habitat est déterminée par la nature des matériaux, l'approvisionnement en eau et en électricité et les éléments de son environnement naturel, artificiel et hygiénique. A côté de la qualité de l'habitat, on enregistre aussi dans les villes africaines un problème de crise de logement. Voulant saisir ce phénomène à Lubumbashi, l'enquête recherche des données sur le statut d'occupation des ménages, la durée de leur séjour dans la résidence qu'ils occupent et le coût du loyer payé par les ménages qui se logent en qualité de locataire.

\section{Organisation et déroulement de l'enquête}

Avant de procéder au recrutement des enquêteurs, il était nécessaire d'évaluer la tâche à remplir et de définir les conditions permettant de l'exécuter. On devait aussi tenir compte qu'une enquête de ce genre ne pouvait pas prendre trop de temps, certainement pas plus de vingt jours. Comme l'objectif était d'atteindre au moins 15000 ménages, cent enquêteurs étaient en mesure de rencontrer cette demande. Mais au regard du budget, on ne pouvait avoir recours qu'à 85 enquêteurs. Si un enquêteur pouvait remplir quotidiennement dix fiches, c'est-à-dire enquêter auprès de dix ménages, 17000 ménages pouvaient être atteints en vingt jours. Ainsi la tâche journalière de l'enquêteur pouvait être ramenée à moins de dix ménages.

Pour procéder au recrutement, une annonce a été rédigée, remise et diffusée à la radio de l'Université et affichée aux valves du Bâtiment administratif de l'Université de Lubumbashi. Un bureau de recrutement a été installé dans un local du Bâtiment administratif de l'Université. Le critère de base de recrutement était un niveau universitaire d'au moins trois années d'études, c'est-à-dire avoir atteint ou terminé le cycle de graduat. L'offre concernait les candidats de toutes disciplines confondues. Une préférence a été accordée aux étudiants et diplômés des disciplines dont le cursus initie les apprenants aux méthodes et techniques d'enquête de terrain. La maîtrise de la langue swahili, à côté 
de celle du français, a été exigée. De plus, il a été demandé aux experts qui ont participé à diverses enquêtes récemment organisées dans la ville ou dans la province de proposer aussi quelques candidats expérimentés.

Tous les candidats qui se sont fait enregistrer et qui ont répondu aux critères arrêtés, soit près de cent personnes, hommes et femmes confondus, ont été admis à la formation. Il a été estimé que la formation elle-même pouvait être un système de sélection, car c'est au cours de cette session que les candidats se sont rendus compte de l'ampleur de la tâche qui les attendait, avec toutes ses exigences. Le dernier niveau de sélection s'est situé lors de la pré-enquête et aux premiers jours de l'enquête, au cours d'un contrôle minutieux de la tâche remplie.

Les formateurs étaient au nombre de deux : un démographe et un statisticien. Tous deux ont participé à plusieurs enquêtes réalisées à Lubumbashi et dans la Province du Katanga. Ils ont, en outre, contribué à la conception scientifique du dernier recensement de la ville de Lubumbashi.

Prévue pour un jour, la formation s'est faite en deux jours afin de bien indiquer les différentes opérations de l'enquête. La formation a consisté dans un premier temps à donner aux enquêteurs les objectifs assignés au travail qu'ils seront appelés à exécuter. En deuxième lieu, on leur a enseigné les qualités d'un bon enquêteur et les responsabilités dans un travail de recherche. Par la suite, une longue séance a consisté à la présentation du protocole d'enquête : toutes les variables ont fait l'objet d'un long commentaire et un guide a été remis à chacun, ainsi que le feuillet du protocole. Les formateurs leur ont appris comment remplir chaque case du questionnaire. Une déontologie du questionnement leur a été enseignée. La formation s'est terminée par une séance relative à la procédure à suivre pour faire un relevé parcellaire. En principe cette tâche revenait à la commission préparatoire, mais il s'est avéré qu'elle conviendrait mieux à ceux qui exécuteront le travail de terrain. Le relevé parcellaire leur a permis de reconnaître à l'avance le milieu et de se familiariser déjà par rapport au terrain. Les formateurs ont signalé le fait qu'au Congo il n'existe pas de listes exhaustives de numéros parcellaires, ils ont montré toutes les difficultés liées à l'identification parcellaire tant dans les quartiers urbanisés que dans ceux non planifiés.

La formation des enquêteurs s'est terminée par une « descente sur le terrain » car il fallait reconnaître le milieu avant le travail proprement dit et circonscrire les secteurs d'enquête. C'est à cette occasion que les enquêteurs, encadrés par leurs formateurs et superviseurs, ont procédé au relevé parcellaire. Cette activité a permis aux organisateurs de désigner les parcelles à investiguer, par rue et par quartier. C'était aussi un test d'endurance. Cette étape qui a duré trois jours, du 22 au 24 juillet 2002, a constitué la pré-enquête, car elle a permis aux organisateurs de soumettre déjà le protocole à quelques ménages au choix.

\section{Organisation du travail sur le terrain}

L'implication des autorités administratives tant de l'Université que de la ville s'est concrétisée par la remise d'ordres de mission à tous les enquêteurs et à leurs encadreurs, ordres de mission signés conjointement par le recteur et le maire. Un communiqué de presse a été écrit et mis à la signature du recteur. Envoyé à la radio de l'Université et à la RTNC (Radio et Télévision Nationale Congolaise), ce message a été diffusé chaque jour à trois reprises et cela jusqu'au dernier jour de la récolte des données par les enquêteurs. 
A cette sensibilisation par les médias, il a aussi été fait recours à la sensibilisation directe au moyen d'un mégaphone monté sur un véhicule pour informer la population de la réalisation de l'enquête. Cette dernière méthode était appliquée dans les quartiers et rues à chaque veille du passage des enquêteurs dans le secteur.

Pour plus d'efficacité et pour mieux assurer la qualité de l'enquête, trois équipes d'enquêteurs avaient été constituées. Chaque équipe, supervisée par un responsable, était orientée vers les quartiers et rues qui avaient fait l'objet d'un travail de relevé parcellaire par la même équipe. En ce qui concerne la prise en charge du déplacement des enquêteurs, quatre mini-bus ont été loués et mis à leur disposition. Pour ce faire, tous les enquêteurs étaient tenus d'arriver au bureau central de l'enquête à 7 h30. Là, on procédait au contrôle physique : chacun devant son encadreur recevait éventuellement quelques remarques et observations en rapport avec le travail fait la veille et c'est à cette occasion que la tâche du jour lui était confiée (les fiches d'enquête et les numéros des parcelles à visiter). L'embarquement dans le bus se faisait autour de $8 \mathrm{~h} 00$. A partir de $16 \mathrm{~h} 00$, les bus passaient pour les reconduire chez eux.

Pour assurer un minimum d'organisation sur le terrain d'enquête et dans l'objectif de guider les mouvements de l'enquêteur, on remettait, au départ du Bureau Central de l'Enquête, à chaque enquêteur un jeton portant le nom de l'avenue et les numéros des parcelles à visiter cette journée-là. En plus, sur le terrain, un endroit était chaque fois choisi comme bureau d'antenne : là, le superviseur, après avoir placé les enquêteurs sur les avenues, se tenait pour recevoir les doléances de ces derniers, les guider en attirant leur attention par des petites remarques et observations en cas d'un mauvais remplissage de fiches. Dans les cas où il s'avérait que quelques fiches n'étaient pas bien remplies, le superviseur avait l'obligation de les renvoyer dans ces ménages pour compléter ou corriger une information mal enregistrée.

Bien que le début ait été un peu difficile, lorsque les enquêteurs n'étaient pas encore habitués aux codes de réponse, la suite du travail a été abordée avec compétence. Finalement, non sans peine, après vingt jours, la fin du périple a donné satisfaction à l'ensemble des intervenants. Cependant, il fallait en plus un travail complémentaire, nécessaire pour combler quelques oublis constatés çà et là. A cet effet, deux journées supplémentaires ont été consacrées pour les parcelles qui n'avaient pas été atteintes lors du premier passage. Dix enquêteurs choisis parmi les meilleurs se sont attelés à cette tâche. Finalement, l'enquête proprement dite a duré vingt-deux jours, c'est-à-dire du 29 juillet au 22 août 2002 .

\section{Encodage}

La difficulté principale de l'encodage venait de la nécessité d'une structure multirelationnelle pour la base de données: chaque ménage avait à la fois ses propres caractéristiques, communes à tous ses membres, mais ces derniers possédaient eux-mêmes des caractéristiques différenciées (par exemple, pour la fécondité ou la mortalité infantile). Il fallait donc gérer plusieurs bases de données simultanément : celle des ménages, celle des individus et celle de la fécondité. Et chaque base devait être reliée aux autres.

Le modèle d'encodage a été conçu par une équipe d'informaticiens de l'Université de Lubumbashi au cours d'un stage aux Facultés Universitaires Notre-Dame de la Paix de Namur en Belgique. Ces derniers ont conçu un programme que devaient exécuter les préposés à l'encodage. Ce programme, écrit en langage « Php », permettait aux encodeurs 
de retrouver un environnement semblable aux feuilles d'enquête. Les travaux de dépouillement ont pris trois mois, d'avril à juin 2003, au lieu d'un seul mois initialement prévu. Il faut dire que le volume du travail à réaliser avait été sous-estimé au départ. Il en est de même du nombre d'encodeurs : au lieu de trois encodeurs envisagés par les organisateurs, finalement sept personnes se sont attelées à la tâche. Malgré cela, un travail de vérification, de nettoyage et d'harmonisation de la base de données s'est poursuivi au-delà de la période susmentionnée. A l'issue de l'encodage, la base informatique des données recueillies contient plus de 130000 lignes de données, chaque ligne ayant environ 60 variables. Cela représente l'équivalent de 70 Megaoctets de données.

\section{L'analyse statistique}

C'est lors d'un séjour d'un mois en Belgique que les deux responsables de l'enquête, ainsi que le statisticien responsable de l'encodage, Augustin Kanyimbu, ont rédigé l'analyse statistique et socio-économique des données de l'enquête. Vu l'importance de la base de données, et le peu de temps disponible, seules des statistiques descriptives ont pu être mises en évidence. Cela peut sembler maigre comme résultat, mais il faut savoir que la manipulation d'une telle masse de données demande une maîtrise (longue à acquérir) d'un langage d'interrogation de base de données (ici, le langage SQL) afin de nettoyer la base des données erronées et d'obtenir des statistiques croisées. Un travail d'analyse statistique plus fine aurait du avoir lieu, mais cela nécessitait l'apprentissage de techniques et de logiciels statistiques complexes. Pour le moment, nous ne savons pas si des chercheurs congolais, étudiants ou doctorants, ont l'intention d'utiliser la base de données qui se trouve sur le net afin d'en tirer des conclusions novatrices et utiles pour les décideurs socio-économiques ou politiques.

\section{Difficultés rencontrées}

\section{La connectivité des recherches}

Comme nous le disions dans l'introduction, le grand défi d'une telle recherche est la disproportion entre les attentes des chercheurs (valider un recensement et avoir une image socio-économique fine des ménages Lushois) et les moyens disponibles en termes financiers, de temps de travail et d'expertise.

L'étape d'échantillonnage des ménages et des parcelles n'a pas posé de véritable problème, vu l'expérience acquise par les responsables lors d'enquêtes plus anciennes. Evidemment, d'autres techniques plus sophistiquées d'échantillonnage auraient pu être utilisées (sur-pondération de certains quartiers ou type de ménage, en fonction de la représentativité dans le recensement). Mais cela aurait nécessité un travail de recherche et d'analyse du recensement, à réaliser avant l'enquête, ce qui n'a pu se faire vu la difficulté d'accès aux résultats détaillés du recensement (base des données informatisée). Ce point pose le problème général de la connectivité des recherches.

Que ce soit lors de la préparation de l'enquête (rédaction du questionnaire, choix des thèmes de l'enquête) ou lors de la rédaction des résultats, les liens avec les autres recherches connexes furent difficiles à établir. Par exemple, le choix des variables a rarement été justifié par une étude de la littérature afin de pouvoir comparer les résultats de notre enquête avec les études récentes. Ceci est compréhensible car, pour que les autres recherches interagissent avec la recherche en cours, il faut absolument que ces premières soient publiées ou facilement accessibles, mais aussi que les responsables aient 
le temps de se former et de se mettre à jour, en termes de bibliographie. On perçoit, en lisant les bibliographies des articles publiés, le décalage des recherches congolaises. Heureusement, l'accès à internet est en train de se diffuser dans les universités, ce qui permet aux chercheurs et aux étudiants de faire un travail de mise à jour sur tel ou tel sujet.

\section{La déontologie de la recherche}

Lors de la rédaction du questionnaire, plusieurs difficultés ont été soulevées par le bailleur de fonds, entre autres, concernant la mention de l'origine (chefferie) des personnes ainsi que du respect de l'anonymat des réponses. Il est clair que l'encodage de toutes les données recueillies présente un danger en termes de respect de la vie privée. L'outil informatique permet de sélectionner facilement telle ou telle catégorie de personnes. Cependant, il est impossible de ne pas conserver dans les questionnaires des informations permettant l'identification d'un individu, au risque de ne pouvoir contrôler la véracité des réponses. Toute enquête de ce type doit se soumettre à des règles déontologiques de respect de la vie privée.

Le choix des responsables de l'enquête a été de ne pas encoder les données dites « sensibles », mais de les garder dans le questionnaire, pour un décompte manuel futur. Le non-encodage interdisait toute malversation de données, qu'on désirait, par ailleurs, diffuser très largement. Par contre, les archives " papier» de l'enquête sont accessibles à des chercheurs bien identifiés, et ayant clairement conscience de leurs obligations déontologiques. En plus de l'ampleur d'un tel travail de dépouillement, toute mention des noms et prénoms a été supprimé dans la version « papier » (elle ne se trouve évidemment pas non plus sur le support informatique).

\section{La technicité des analyses}

Un problème important de ce genre de recherche est la disponibilité des chercheurs et des responsables pour faire un véritable travail de recherche, en profondeur. Un tel travail devrait être réalisé par un ou plusieurs doctorants, dont c'est l'activité quasiment exclusive. Or, la situation financière des universités congolaises ne permet pas un tel investissement sur un long terme. D'où un manque de rigueur ou d'approfondissement dans l'analyse. Cela se ressent en particulier dans l'absence de techniques plus sophistiquées d'analyse statistique. Tout un travail de corrélation et de prédiction aurait été le bienvenu. De même, lorsque des résultats semblent erronés, il aurait fallu procéder à une nouvelle vérification en profondeur de l'encodage, mais le temps manqua. Ce fut par exemple le cas d'un mauvais encodage systématique de la part du revenu affecté à telle ou telle activité : par suite d'une erreur informatique, toutes les données étaient mises à zéro. Devoir reprendre les 13000 questionnaires pour cette seule question n'a pas été possible.

Ce point montre l'importance, tout comme le premier point, d'une formation des intervenants et surtout de leur « disponibilisation » pour un tel travail. Il faut accepter de « perdre du temps » à tester des hypothèses, à essayer des techniques nouvelles. Mais cela coûte, et souvent les bailleurs de fonds désirent des résultats rapidement. 


\section{Conclusions}

En conclusion, on peut affirmer que de telles recherches sont vitales pour les universités congolaises. Grâce à elles, les départements sont revitalisés et de nouveaux savoirs sont appréhendés. Cependant, l'expérience montre combien la réalité difficile du contexte local oblige les responsables de ces recherches à faire l'impasse sur telle ou telle étape méthodologique, par manque de finances, de temps ou d'expertise. Ces lacunes ne doivent pas être occultées, mais plutôt signalées afin qu'un travail de recherche en seconde lecture puissent être réalisé pour les réduire.

Pour cela, la mise à disposition des données de base, sous forme informatique par exemple, est essentielle et assez aisée à faire. De même, nous plaidons pour que soient également disponibles les études antérieures. Si la contextualisation locale des recherches nous semble souvent effective, il n'en est cependant pas de même de l'insertion de ces recherches au niveau de la communauté universitaire en général. Le désenclavement de la recherche congolaise passe par le respect des exigences de qualité telles que l'articulation avec les recherches existantes, la formation aux techniques avancées, l'approfondissement de questions plus théoriques, la publication des résultats. Cela ne sera possible que dans un cadre de travail serein où du temps est libéré pour les recherches de longue haleine.

\section{Références bibliographiques}

Houyoux, J. et Y. Leconnet, 1975. Lubumbashi. Démographie, budgets ménagers et étude du site. Kinshasa : Bureau d'études d'aménagement urbain.

Institut National des Statistiques

1989. Enquête budgets ménagers : ville de Lubumbashi 1985. Kinshasa.

1993. Recensement scientifique de la population 1984. Projections démographiques. Zaïre et régions 1984-2000. Kinshasa.

Kakoma Sakatolo Zambeze (éd.), 2002. Le profil sanitaire du Lushois, rapport de recherches effectuées durant la troisième session des travaux de l'Observatoire de changement urbain en février-juin 2001. Université de Lubumbashi.

Petit, Pierre (éd.), 2003. Ménages de Lubumbashi entre précarité et recomposition. Paris : L’Harmattan. 


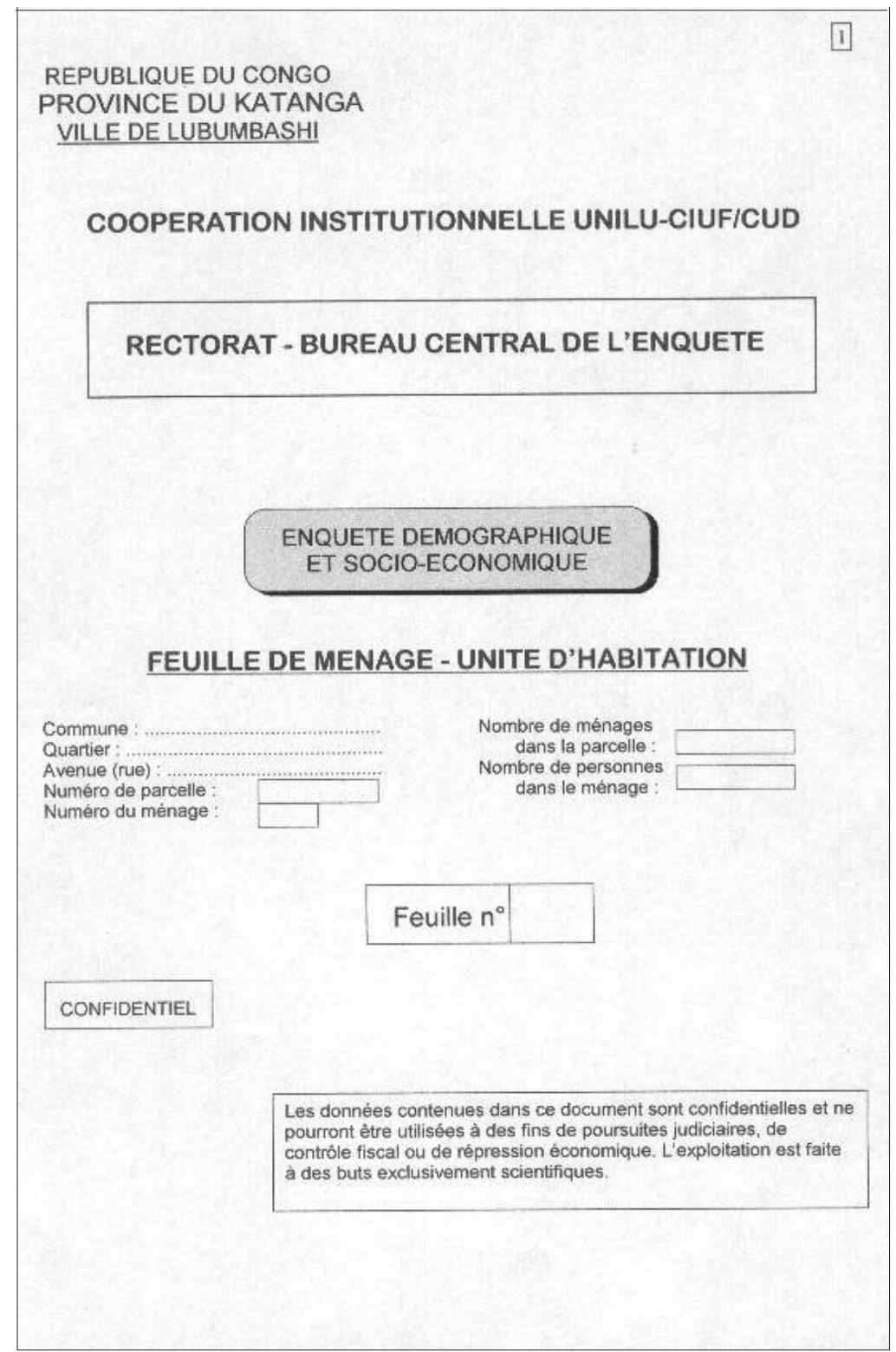




\section{LANGUES PARLEES}

29. Indiquez ci-dessus les langues nationales et ètrangères parlées dans votre ménage :

Kikongo
Lingala
Swahili
Tshiluba

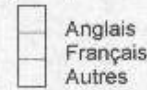

\section{REVENU DU MENAGE}

30. Quelle est la source principale de revenu dans le ménage ?

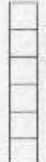

le salaire

le commerce avec registre de commerce

le petit commerce

I'agriculture

les activités libérales (cabinets d'avocats, médecins oeuvrant en privé, bureaux d'études) autres activités

31. Quelies sont les voies principales d'approvisionnement en denrées alimentaires?

achat agriculture familiale aides de proches autres

32. En cas de difficultés matérielles, financières ou alimentaires, indiquez la personne à laquelle vous vous référez prioritairement pour recevoir des aides:

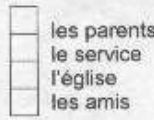

33. Possédez-vous dans votre ménage :

\begin{tabular}{|l} 
une radio \\
une télévision \\
une vidéo \\
une antenne parabolique \\
un congélateur \\
une bicyclette
\end{tabular}

34. Recevez-vous un salaire :

$\square$ régulièrement
irrégulièrement
Ne sait pas

35. Comment utilisez-vous votre salaire?

$$
\begin{aligned}
& \text { Ration alimentaire } \\
& \text { Tontine (kinkulimba) } \\
& \text { Fonds de commerce } \\
& \text { Achat des biens personnels }
\end{aligned}
$$

les voisins

les mutuelles tribales ou régionales à personne

autres

\section{une mobilette}

une voiture

une camionnette

une cuisinière

une maison

autres biens 